\title{
Condensation Energy for a Two-Gap Superconducting State in Nanoparticles
}

\author{
Sergei P. Kruchinin, ${ }^{1}$ Hiroyuki Kawabe, ${ }^{2}$ Hidemi Nagao, ${ }^{3}$ and Yasuhiro Nakazawa ${ }^{4}$ \\ ${ }^{1}$ Bogolyubov Institute for Theoretical Physics, National Academy of Science of Ukraine, Kiev 03680, Ukraine \\ ${ }^{2}$ Department of Social Work, Faculty of Social Work, Kinjo University, 1200 Kasama, Hakusan, Ishikawa 924-8511, Japan \\ ${ }^{3}$ Division of Mathematical and Physical Science, Graduate School of Natural Science and Technology, Kanazawa University, \\ Kakuma, Kanazawa 920-1192, Japan \\ ${ }^{4}$ Department of Chemistry, Graduate School of Science, Osaka University, Toyonaka, Osaka 560-0043, Japan
}

Correspondence should be addressed to Sergei P. Kruchinin; skruchin@i.com.ua

Received 12 March 2013; Revised 2 June 2013; Accepted 6 June 2013

Academic Editor: Raphael Schneider

Copyright (C) 2013 Sergei P. Kruchinin et al. This is an open access article distributed under the Creative Commons Attribution License, which permits unrestricted use, distribution, and reproduction in any medium, provided the original work is properly cited.

\begin{abstract}
Recent technology and experiments have fabricated high-quality superconducting $\mathrm{MgB}_{2}$ nanoparticles. We investigate properties of two-gap superconductivity in nanosized systems by using a two-sublevel model. In the present work, we analyze the results obtained for superconducting granules in the case of multiband superconductivity. We discuss the finite size effect in multiband superconductors. A definition of the critical level spacing of two-gap superconductivity is also presented, and we discuss the condensation energy and the parity gap of two-gap superconductivity in relation to the size dependence of those properties with two bulk gaps and the effective pair scattering process between two sublevels.
\end{abstract}

\section{Introduction}

Recent advances in nanoscience have demonstrated that fundamentally new physical phenomena are found when systems are reduced in size to dimensions that become comparable to the fundamental microscopic length scales of a material under study. Superconductivity is a macroscopic quantum phenomenon, and therefore, it is especially interesting to see how this quantum state is influenced when the samples are reduced to nanometer sizes. In such systems, new states of matter can be engineered that do not occur in bulk materials.

The properties of superconducting materials with ultrasmall sizes differ from those in bulk [1]. The characteristics of a superconducting granule depend on the discrete energy spectrum, the parity of the number of electrons, and quantum fluctuations [2]. The dependence of superconducting properties on the parity of the number of electrons participating in the Cooper coupling was called the "parity effect" [3]. The analogous phenomenon is known in nuclear physics. The parity effect was experimentally observed in small superconducting aluminum granules $[4,5]$.
After the discovery of multiband superconductors such as $\mathrm{MgB}_{2}$ with $T_{c}=39 \mathrm{~K}[6]$ and iron pnictides with $T_{c}=$ $45 \mathrm{~K}$ [7], the physicists were faced with the question about the influence of the number of bands on the properties of superconducting granules. This problem was discussed in recent works [8-11]. Here, we plan to generalize the results of calculations of the thermodynamics of superconducting granules to the case of a two-band model of superconductivity.

Recent discovery of superconductivity of $\mathrm{MgB}_{2}$ has much attracted great interest with multigap superconductivity [6]. Although multigap superconductivity had been discussed theoretically [12] in 1958, the multigap superconductivity has been observed experimentally in the 1980s [13].

$\mathrm{MgB}_{2}$ is the first material whose effects are so dominant and implications are so thoroughly explored. Magnesium diboride is reported to be an anisotropic superconductor with conventional Bardeen-Cooper-Schrieffer electron-phonon coupling $[7,14]$. The band structure of $\mathrm{MgB}_{2}$ calculated in several works since the discovery of the superconductivity 
is similar to that of graphite and is formed by by the $\sigma$ and $\pi$ zones. Magnesium diboride has two superconducting gaps, $4 \mathrm{meV}$ and $7.5 \mathrm{meV}$, due to the $\pi$ and $\sigma$ electron bands. The two-gap structure was established in a number of experiments, and the two-gap superconductivity has been also discussed by many groups.

In the recent years, great efforts have been devoted to the fabrication of $\mathrm{MgB}_{2}$ nanostructures that could play a crucial role in the field of applied superconductivity [7]. The ideal candidate is one-dimensional (1D) nanostructures including nanotubes, nanowires, and nanoparticles. As for $\mathrm{MgB}_{2}$, nanoparticles of approximately $20-100 \mathrm{~nm}$ in size are available (see Figure 1) [8].

For ultra-small superconducting grains, the experiments $[4,5]$ by Black et al. have also generated much interest in the size dependence of superconductivity. Properties of ultra-small superconducting grains have been theoretically investigated by many groups $[10,11,15-20]$. Such ultrasmall grains were considered by Anderson [1]. The standard BCS theory gives a good description of the phenomenon of superconductivity in large samples. For ultra-small Al grains $[4,5]$, the bulk gap has been discussed in relation to physical properties of ultra-small grains such as the parity gap [3], condensation energy [18], electron correlation [16], and as well as the size dependence of the level spacing [17] of samples. In this paper, we investigate the properties of two-gap superconductivity in a nanosized system by using a two-sublevel model in the case of strong interaction. We discuss the condensation energy and the parity gap of twogap superconductivity in relation to the size dependence of those properties in the case of two bulk gaps and the effective pair scattering process between two sublevels.

\section{The Model and the Solution}

In the case of a two-gap superconductor, we can consider a model with two sublevels corresponding to two independent bands. We consider a pairing Hamiltonian with two sublevels $\nu=1,2$ corresponding to two bands 1 and 2 written as

$$
H=H_{0}+H_{\text {int }},
$$

where

$$
\begin{gathered}
H_{0}=\sum_{j, \sigma, v}\left[\varepsilon_{v j}-\mu\right] a_{v j \sigma}^{\dagger} a_{v j \sigma}, \\
H_{\text {int }}=-\sum_{\nu} g_{\nu} \sum_{j, j^{\prime} \in I_{\nu}} a_{v j \uparrow}^{\dagger} a_{v j \downarrow}^{\dagger} a_{v j^{\prime} \downarrow} a_{v j^{\prime} \uparrow} \\
+g_{12} \sum_{j \in I_{1}, k \in I_{2}} a_{1 j \uparrow}^{\dagger} a_{1 j \downarrow}^{\dagger} a_{2 k \downarrow} a_{2 k \uparrow}+\text { H.c. }
\end{gathered}
$$

where $a_{v j \sigma}^{\dagger}\left(a_{v j \sigma}\right)$ is the creation (annihilation) operator in sublevels $v$ with spin $\sigma$ and the energies $\varepsilon_{v j}$. Here, the operators for each sublevel satisfy the anticommutation relations, the operators between sublevels are independent, and $\mu$ is the chemical potential. The sums of $j$ and $k$ in (3) are over the set $I_{1}$ of $N_{1 I_{1}}$ states corresponding to a halffilled band 1 with fixed width $2 \omega_{1 D}$ and the set $I_{2}$ of $N_{2 I_{2}}$

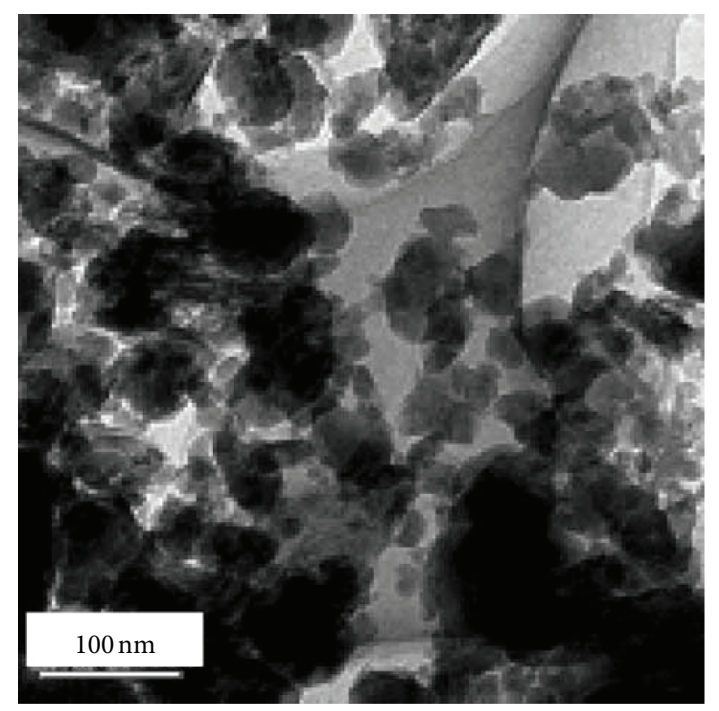

FIGURE 1: The micrograph of 20-100 nm nanoparticles of $\mathrm{MgB}_{2}$.

states for band 2, respectively. The second term in (1) is the interaction Hamiltonian, $g_{1}$ and $g_{2}$ are the effective electron-electron interaction constants including the e-p interaction for each sublevel, respectively, and $g_{12}$ is the effective interaction constant that corresponds to the pair scattering process including the phonon scattering between two bands. Note that, in the bulk limit of (1), we can derive a similar coupled gap equation presented in [9].

In this study, we assume that the Debye energies for two sublevels are the same $\omega_{1 D}=\omega_{2 D}=\omega_{D}$. Within this assumption, $N_{1 I_{1}}$ and $N_{2 I_{2}}$ are relatively estimated by the density of state (DOS) for two bands as $N_{1 I_{1}} / N_{2 I_{2}}=\rho_{1} / \rho_{2}$, where $\rho_{1}$ and $\rho_{2}$ are DOSs for two bands, respectively. The interaction constants $g_{1}$ and $g_{2}$ can be written as $d_{1} \lambda_{1}$ and $d_{2} \lambda_{2}$, respectively, $d_{1}=2 \omega_{D} / N_{1 I_{1}}$ and $d_{2}=2 \omega_{D} / N_{2 I_{2}}$ mean the mean energy level spacing, and $\lambda_{1}$ and $\lambda_{2}$ are the dimensionless parameters for two sublevels. We consider the intersublevel interaction constant $g_{12}=\sqrt{d_{1} d_{2}} \lambda_{12}$. In summary, we obtain a relation of $\rho_{1} / \rho_{2}=N_{1 I_{1}} / N_{2 I_{2}}=d_{2} / d_{1}$. It is convenient to apply the path integral approach to the treatment of fluctuations of the order parameters. In works $[16,17]$, the problem of ultra-small superconducting grains was considered within the path integral method

$$
Z(\mu, T)=\int D^{2} \Delta(\tau) e^{-S[\Delta]}
$$

where $T$ is the temperature and $S[\Delta]$ is the action.

We now adapt the developed technique of path integration in the case of two-gap superconductivity. In what follows, we will use this method to study the influence of fluctuations on the two-gap superconductivity in ultra-small superconducting grains. This approach gives an exact expression for the canonical partition function of a superconductor [3]. Within the path integral approach, we obtain the canonical 
partition function for the Hamiltonian of (1) in the following form:

$$
Z(\mu, T)=\int D \Delta_{1} D \Delta_{1}^{*} D \Delta_{2} D \Delta_{2}^{*} e^{-S\left[\Delta_{1}, \Delta_{2}\right]},
$$

where the action $S\left[\Delta_{1}, \Delta_{2}\right]$ is defined as

$$
\begin{aligned}
& S\left[\Delta_{1}, \Delta_{2}\right] \\
& =-\sum_{j}\left[\operatorname{Tr} \ln G_{1 j}^{-1}-\frac{\xi_{1 j}}{T}\right]-\sum_{k}\left[\operatorname{Tr} \ln G_{2 k}^{-1}-\frac{\xi_{2 k}}{T}\right] \\
& +\int_{0}^{1 / T} d \tau \frac{1}{g_{1} g_{2}-g_{12}^{2}} \\
& \quad \times\left[g_{2}\left|\Delta_{1}(\tau)\right|^{2}+g_{1}\left|\Delta_{2}(\tau)\right|^{2}\right. \\
& \left.\quad+g_{12}\left(\Delta_{1}(\tau) \Delta_{2}(\tau)^{*}+\Delta_{1}(\tau)^{*} \Delta_{2}(\tau)\right)\right] .
\end{aligned}
$$

Here, $\Delta_{1}$ and $\Delta_{2}$ are bulk gaps for sublevels 1 and 2, respectively, $\xi_{v j}=\varepsilon_{v j}-\mu$, and $G_{v j}^{-1}\left(\tau, \tau^{\prime}\right)=\left[-d / d \tau-\xi_{v j} \sigma^{z}-\right.$ $\left.\Delta_{v}(\tau) \sigma^{+}-\Delta_{\nu}^{*}(\tau) \sigma^{-}\right] \delta\left(\tau-\tau^{\prime}\right)$, where $\sigma^{ \pm}=\sigma^{x} \pm i \sigma^{y}$ and $\sigma^{x, y, z}$ are the Pauli matrices. $G_{1}^{-1}$ and $G_{2}^{-1}$ satisfy antiperiodic boundary conditions.

In the case of stronger interaction, $\Delta_{1} \gg d_{1}$ and $\Delta_{2} \gg$ $d_{2}$ can be considered in the mean-field approximation for the order parameters within the path integral approach. Substituting the time-independent order parameters into the action of (6), we have

$$
\begin{aligned}
\Omega(\mu)= & \sum_{j, v}\left(\xi_{v j}-\epsilon_{\nu j}\right)+\frac{1}{g_{1} g_{2}-g_{12}^{2}} \\
& \times\left[g_{2} \Delta_{1}^{2}+g_{1} \Delta_{2}^{2}+g_{12}\left(\Delta_{1}^{*} \Delta_{2}+\Delta_{1} \Delta_{2}^{*}\right)\right],
\end{aligned}
$$

where $\epsilon_{1 j}=\left(\xi_{1 j}^{2}+\Delta_{1}^{2}\right)^{1 / 2}$ and $\epsilon_{2 k}=\left(\xi_{2 k}^{2}+\Delta_{2}^{2}\right)^{1 / 2}$. In (7), the values of $\Delta_{1}$ and $\Delta_{2}$ must be chosen in a way that minimizes $\Omega$. From the minimization of $\Omega$, we obtain the coupled gap equation at zero temperature for a two-gap system:

$$
\left(\begin{array}{c}
\Delta_{1} \\
\Delta_{2}
\end{array}\right)=\left(\begin{array}{cc}
g_{1} \sum_{j} \frac{1}{2 \epsilon_{1 j}} & -g_{12} \sum_{k} \frac{1}{2 \epsilon_{2 k}} \\
-g_{12} \sum_{j} \frac{1}{2 \epsilon_{1 j}} & g_{2} \sum_{k} \frac{1}{2 \epsilon_{2 k}}
\end{array}\right)\left(\begin{array}{c}
\Delta_{1} \\
\Delta_{2}
\end{array}\right) .
$$

From this equation, we formally obtain an expression for a bulk gap at zero temperature in the case of two-gap superconductivity:

$$
\widetilde{\Delta}_{1}=\omega \sinh ^{-1}\left(\frac{1}{\eta_{1}}\right), \quad \widetilde{\Delta}_{2}=\omega \sinh ^{-1}\left(\frac{1}{\eta_{2}}\right),
$$

where $1 / \eta_{v}=\left(\lambda_{v^{\prime}}+\alpha_{ \pm}^{(-1)^{\nu^{\prime}}}\left[\eta_{1}, \eta_{2}\right] \lambda_{12}\right) /\left(\lambda_{1} \lambda_{2}-\lambda_{12}^{2}\right)\left(\nu \neq \nu^{\prime}\right)$ and $\alpha_{ \pm}\left[\eta_{1}, \eta_{2}\right]= \pm \sinh \left(1 / \eta_{1}\right) / \sinh \left(1 / \eta_{2}\right)$. For two-band superconductivity, we can consider two cases for the phases of the gaps: $\operatorname{sgn}\left(\widetilde{\Delta}_{1}\right)=\operatorname{sgn}\left(\widetilde{\Delta}_{2}\right)$ and $\operatorname{sgn}\left(\widetilde{\Delta}_{1}\right)=-\operatorname{sgn}\left(\widetilde{\Delta}_{2}\right)$. For the same phase, $\alpha_{+}$is used in (8), and we use $\alpha_{-}$for the opposite phases. Note that $\widetilde{\Delta}_{1}=-\widetilde{\Delta}_{2}$ in the limit of strong intersublevel coupling $\lambda_{12}$, that is, for the opposite phases. At $\lambda_{12}=0$, we find the same results of two bulk gaps derived from the conventional BCS theory for two independent sublevels.

In nanosized single-band superconductivity, the condensation energy can be defined as $E_{N, b}^{C}(\lambda)=E_{N, b}^{G}(0)-$ $E_{N, b}^{G}(\lambda)-n \lambda d$, where $E_{N, b}^{G}$ is the ground state energy of an $N$-electron system in the interaction band, $b$ is the number of electrons on single occupied levels, and $\lambda$ and $n$ are the dimensionless coupling parameter and the number of pair occupied levels, respectively. In the case of a nanosized two-band system, the condensation energy can be written in a similar way as follows: $E_{N_{1}, b_{1} ; N_{2}, b_{2}}^{C}\left(\lambda_{1}, \lambda_{2}, \lambda_{12}\right)=$ $E_{N_{1}, b_{1} ; N_{2}, b_{2}}^{G}(0,0,0)-E_{N_{1}, b_{1} ; N_{2}, b_{2}}^{G}\left(\lambda_{1}, \lambda_{2}, \lambda_{12}\right)-\sum_{\nu} n_{\nu} \lambda_{\nu} d_{\nu}$, where $E_{N_{1}, b_{1} ; N_{2}, b_{2}}^{G}\left(\lambda_{1}, \lambda_{2}, \lambda_{12}\right)$ means the ground state energy of the $\left(N_{1}+N_{2}\right)$-electron system. From (5) and (7), the condensation energy of a two-sublevel system can be expressed in terms of the condensation energies of independent single level systems:

$$
\begin{aligned}
& E_{N_{1}, b_{1} ; N_{2}, b_{2}}^{C}\left(\lambda_{1}, \lambda_{2}, \lambda_{12}\right) \\
& =E_{N_{1}, b_{1}}^{C}\left(\lambda_{1}\right)+E_{N_{2}, b_{2}}^{C}\left(\lambda_{2}\right) \\
& \quad-\frac{\lambda_{12}^{2}}{\lambda_{1} \lambda_{2}-\lambda_{12}^{2}}\left(\frac{\Delta_{1}^{2}}{d_{1} \lambda_{1}}+\frac{\Delta_{2}^{2}}{d_{2} \lambda_{2}}+\frac{2\left(\Delta_{1}^{*} \Delta_{2}+\Delta_{1} \Delta_{2}^{*}\right)}{\sqrt{d_{1} d_{2}} \lambda_{12}}\right),
\end{aligned}
$$

where $E_{N_{1}, b_{1}}^{C}\left(\lambda_{1}\right)$ and $E_{N_{2}, b_{2}}^{C}\left(\lambda_{2}\right)$ correspond to the condensation energies for single band cases and the third term means the cross term between two bands. At the same phases of $\Delta_{1}$ and $\Delta_{2}$, the condensation energy of (10) decreases; that is, there appears the instability by the coupling constant $\lambda_{12}$. On the other hand, at the opposite phases, the condensation energy becomes larger, because $\Delta_{1}^{*} \Delta_{2}+\Delta_{1} \Delta_{2}^{*}<0$. We can expect that the condensation energy of two-gap superconductivity becomes more stable than that of two independent systems due to the intersublevel coupling $\lambda_{12}$ and the opposite phases.

To discuss the critical level spacing for a two-gap system, we start from the coupled gap equation of (8). For the case of the critical level spacing of a two-gap system, we have

$$
\begin{aligned}
1= & \lambda_{1} \sum_{j} \frac{1}{2\left|\tilde{\xi}_{1 j}\right|}+\lambda_{2} \sum_{k} \frac{1}{2\left|\widetilde{\xi}_{2 k}\right|} \\
& -\left(\lambda_{1} \lambda_{2}-\lambda_{12}^{2}\right) \sum_{j} \frac{1}{2\left|\widetilde{\xi}_{1 j}\right|} \sum_{k} \frac{1}{2\left|\tilde{\xi}_{2 k}\right|},
\end{aligned}
$$

where $\tilde{\xi}_{i}=\xi_{i} / d_{i}$ for sublevels $i=1,2$. For the odd or even grain, (11) can be approximately solved by using the digamma function: for the odd case, the critical level spacing becomes

$$
d_{1 c}^{o}=\omega_{D} e^{\gamma} \exp \left[-\frac{1}{\lambda}\right], \quad d_{2 c}^{o}=\frac{d_{2}}{d_{1}} d_{1 c}^{o},
$$


and, for the even case,

$$
d_{1 c}^{e}=4 \omega_{D} e^{\gamma} \exp \left[-\frac{1}{\lambda}\right], \quad d_{2 c}^{e}=\frac{d_{2}}{d_{1}} d_{1 c}^{e} .
$$

Here, we use

$$
\frac{1}{\lambda}=\frac{1}{2 x}\left[\lambda_{1}+\lambda_{2}-a x+\sqrt{\left(\lambda_{1}-\lambda_{2}-a x\right)^{2}+4 \lambda_{12}^{2}}\right]
$$

with $x=\lambda_{1} \lambda_{2}-\lambda_{12}^{2}, a=\log \left(d_{1} / d_{2}\right)$. From these expressions, we find the relations

$$
\begin{gathered}
d_{1 c}^{e}=4 d_{1 c}^{o}, \quad d_{2 c}^{e}=4 d_{2 c}^{o}, \\
d_{1 / 2 c}^{o} \approx \frac{e^{\gamma}}{2} \exp \left[\frac{1}{\eta_{1 / 2}}-\frac{1}{\lambda}\right] \widetilde{\Delta}_{1 / 2} .
\end{gathered}
$$

In the case of $\left|\lambda_{1}-\lambda_{2}\right| \gg \lambda_{12}$, (16) can be approximately rewritten as

$$
d_{1 / 2 c}^{o} \approx \frac{e^{\gamma}}{2} \exp \left[\frac{\lambda_{2}-\lambda_{1}+2 \alpha \lambda_{12}}{\lambda_{1} \lambda_{2}-\lambda_{12}^{2}}\right] \widetilde{\Delta}_{1 / 2} .
$$

On the other hand, in the limit of $\left|\lambda_{1}-\lambda_{2}\right|=\lambda_{12}$, we have

$$
d_{1 / 2 c}^{o} \approx \frac{e^{\gamma}}{2} \exp \left[\frac{(1+\alpha) \lambda_{12}}{\lambda_{1} \lambda_{2}-\lambda_{12}^{2}}\right] \widetilde{\Delta}_{1 / 2}
$$

For the case of $\lambda_{12}=0$, (16) can be rewritten as $d_{1 / 2 c}^{o} \approx$ $\exp [\gamma] / 2 \exp \left[1 / \lambda_{1}-1 / \lambda_{2}\right] \widetilde{\Delta}_{1 / 2}$. Therefore, when the coupling constants $\lambda_{1}$ and $\lambda_{2}$ become the same value, we have a relation similar to that for a single level system: $d_{1 / 2 c}^{o} \approx 0.89 \widetilde{\Delta}_{1 / 2}$. These results suggest that the critical level spacing strongly depends on $\lambda_{12}$ and the difference between the effective interaction constants for the sublevels. The relation in (15) is the same relation as in the conventional nanosized BCS theory.

In the case of two sublevel spacings, the chemical potential lies halfway between the highest occupied and the lowest unoccupied levels with smaller level spacing in the half-filled case as shown in Figure 2(a). We assume that $d_{1}<d_{2}$ and that the numbers of occupied levels corresponding to each sublevel are $n_{1}$ and $n_{2}$, respectively. Then, the total number of electrons becomes $N=2 n_{1}+2 n_{2}$. When we consider $N=2 n_{1}+2 n_{2}+1$, the chemical potential lies on the level $\varepsilon_{1 n_{1}+1}$, as shown in Figure 2(b). Figure 2(c) shows the position of the chemical potential in the case of $N=2 n_{1}+2 n_{2}+2$. The parity gap of nanosized two-gap superconductivity is written as

$$
\Delta_{p}^{1}=E_{2 n_{1}+1+2 n_{2}, 1}^{G}-\frac{1}{2}\left(E_{2 n_{1}+2 n_{2}, 0}^{G}+E_{2\left(n_{1}+1\right)+2 n_{2}, 0}^{G}\right) .
$$

From (7) and the ground state energy $E_{N, b}^{G}=\Omega_{\mu_{N}}+\mu_{N} N$, we obtain

$$
\Delta_{p}^{1}=\Delta_{1}-\frac{d_{1}}{4}\left(\frac{\rho_{1}}{\rho_{2}}-1\right)
$$

In view of Figures 2(c), 2(d), and 2(e), we can obtain another formula for a parity gap:

$$
\begin{aligned}
\Delta_{p}^{2}= & E_{2\left(n_{1}+1\right)+2 n_{2}+1,1}^{G} \\
& -\frac{1}{2}\left(E_{2\left(n_{1}+1\right)+2 n_{2}, 0}^{G}+E_{2\left(n_{1}+1\right)+2\left(n_{2}+1\right), 0}^{G}\right),
\end{aligned}
$$

which yields

$$
\Delta_{p}^{2}=\Delta_{2}-\frac{d_{2}}{4}\left(\frac{3 \rho_{2}}{\rho_{1}}-1\right) .
$$

The present results suggest two kinds of the dependence of the parity gap on the level spacing. We note that the parity gap does not depend upon the effective interaction $\lambda_{12}$, and the structure around the Fermi level plays an important role, by contributing to the size dependence of the parity gap.

\section{Conclusion}

Thus, we have investigated the properties of nanosized twogap superconductivity by using a two-sublevel model in the framework of the mean-field approximation. In view of the discussion for the condensation energy in nanosized two-gap superconductivity, the phases of the gaps are very important to stabilize the superconductivity. At the same phases, the two-gap superconductivity is instable by the coupling constant $\lambda_{12}$. On the other hand, at the opposite phases, the superconductivity becomes stable. We can expect that the condensation energy of two-gap superconductivity becomes more stable than that of two independent systems due to the intersublevel coupling $\lambda_{12}$ and to the opposite phases.

The parity effect can be observed only at sufficiently low temperatures. It becomes especially significant in ultra-small granules about $10 \AA$ in size. The experimental investigation of superconductivity in such granules becomes possible now due to the developed elegant experimental technique $[6,7]$. The relations concerning the parity effect and the energy condensation in the case of multiband superconductivity, which are obtained in the present work, can be verified in the same experiments as in $[6,7]$ but with the use of nanogranules made of $\mathrm{MgB}_{2}$.

In summary, a model corresponding to nanosized twogap superconductivity has been presented, and the expression of the partition function of a nanosized system has been analytically derived by using the path integral approach. A definition of the critical level spacing of the two-gap superconductivity has been also presented, and we discuss the condensation energy and the parity gap of the two-gap superconductivity in relation to the size dependence of those properties with two bulk gaps and the effective pair scattering process between two sublevels. The results of this work can be tested in the tunneling experiments with $\mathrm{MgB}_{2}$ nanoparticles $[2,4]$. In work [19], the limits of the application of calculations to one-band nanosuperconductors were considered in detail. In the next article, we will plan to study such limits for two-band nanosuperconductors, as well as the influence of fluctuations on the two-gap superconductivity in ultra-small superconducting grains. 


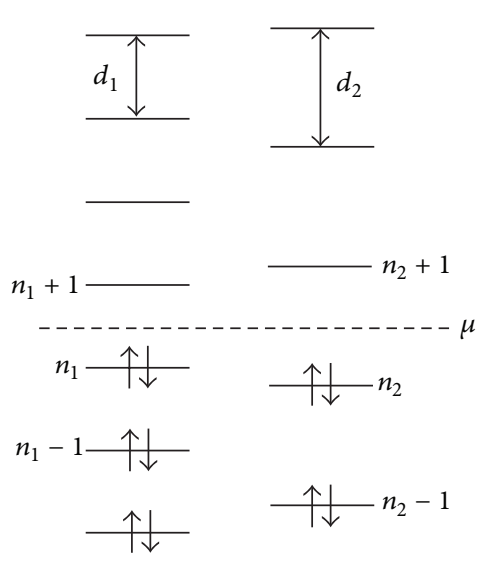

(a)

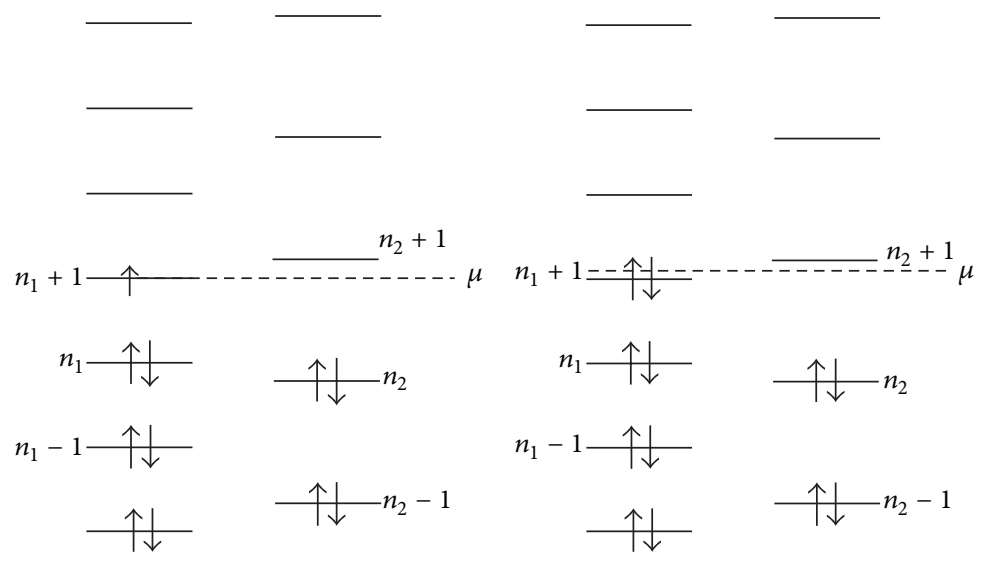

(b) (c)

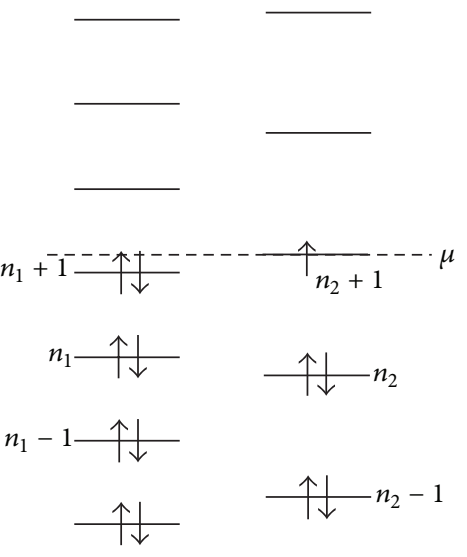

(d)

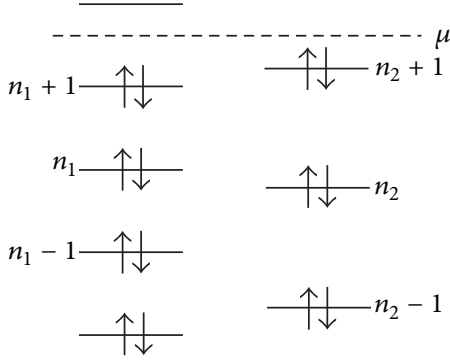

(e)

FIgURE 2: Positioning of the chemical potential relative to the electronic energy levels in a two-gap superconducting grain. Solid and dotted lines mean two sublevels. (a) Half-filled system with $2 n_{1}+2 n_{2}$ electrons, (b) $2 n_{1}+1+2 n_{2}$-electron system, (c) $2\left(n_{1}+1\right)+2 n_{2}$-electron system, (d) $2\left(n_{1}+1\right)+2 n_{2}+1$-electron system, and (e) $2\left(n_{1}+1\right)+2\left(n_{2}+1\right)$-electron system.

\section{Acknowledgments}

The authors thank Professors Jun Akimitsu and Karl Bennemann for their continued encouragement and helpful discussions. S. P. Kruchinin thanks the Research Center for Structural Thermodynamics, Osaka University, for hosting the author as a guest professor to accomplish this work.

\section{References}

[1] P. W. Anderson, "Theory of dirty superconductors," Journal of Physics and Chemistry of Solids, vol. 11, no. 1-2, pp. 28-30, 1959.

[2] J. von Delft and D. C. Ralph, "Spectroscopy of discrete energy levels in ultrasmall metallic grains," Physics Report, vol. 345, no. 2-3, pp. 61-173, 2001.

[3] K. A. Matveev and A. I. Larkin, "Parity effect in ground state energies of ultrasmall superconducting grains," Physical Review Letters, vol. 78, no. 19, pp. 3749-3752, 1997.

[4] C. T. Black, D. C. Ralph, and M. Tinkham, "Spectroscopy of the superconducting gap in individual nanometer-scale aluminum particles," Physical Review Letters, vol. 76, no. 4, pp. 688-691, 1996.
[5] D. C. Ralph, C. T. Black, and M. Tinkham, "Spectroscopic measurements of discrete electronic states in single metal particles," Physical Review Letters, vol. 74, no. 16, pp. 3241-3244, 1995.

[6] P. Shirage, K. Miyazawa, K. Kihou et al., "Synthesis of ErFeAsObased superconductors by the hydrogen doping method," Europhysics Letters, vol. 92, no. 5, p. 57011, 2010.

[7] Q. Yang, J. Sha, X. Ma, Y. Ji, and D. Yang, "Aligned single crystal $\mathrm{MgB}_{2}$ nanowires," Superconductor Science and Technology, vol. 17, no. 8, p. L31, 2004.

[8] C. Cui, D. Liu, Y. Shen et al., "Nanoparticles of the superconductor $\mathrm{MgB}_{2}$ : structural characterization and in situ study of synthesis kinetics," Acta Materialia, vol. 52, no. 20, pp. 57575760, 2004.

[9] H. Nagao, S. P. Kruchinin, A. M. Yaremko, and K. Yamaguchi, "Multiband superconductivity," International Journal of Modern Physics B, vol. 16, no. 23, pp. 3419-3428, 2002.

[10] E. A. Yuzbashyan, A. A. Baytin, and B. L. Altshuller, "Finite-size corrections for the pairing Hamiltonian," Physical Review B, vol. 71, no. 9, Article ID 094505, 10 pages, 2002.

[11] A. M. Garsia-Garsia, J. D. Urbina, E. Yuzbashyan, K. Rihter, and B. Altshuler, "Bardeen-Cooper-Schrieffer theory of finite-size superconducting metallic grains," Physical Review Letters, vol. 100, no. 18, Article ID 187001, 2008. 
[12] H. Suhl, B. T. Matthias, and R. Walker, "Bardeen-CooperSchrieffer theory of superconductivity in the case of overlapping bands," Physical Review Letters, vol. 3, no. 12, pp. 552-554, 1959.

[13] G. Binnig, A. Baratoff, H. E. Hoenig, and J. G. Bednorz, "Twoband superconductivity in $\mathrm{Nb}$-doped $\mathrm{SrTiO}_{3}$," Physical Review Letters, vol. 45, no. 16, pp. 1352-1355, 1980.

[14] J. Nagamatsu, N. Nakagawa, T. Muranaka, Y. Zenitani, and J. Akimitsu, "Superconductivity at $39 \mathrm{~K}$ in magnesium diboride," Nature, vol. 410, no. 6824, pp. 63-64, 2001.

[15] B. Jankó, A. Smith, and V. Ambegaokar, "BCS superconductivity with fixed number parity," Physical Review B, vol. 50, no. 2, pp. 1152-1161, 1994.

[16] J. von Delft, A. D. Zaikin, D. S. Golubev, and W. Tichy, "Parityaffected superconductivity in ultrasmall metallic grains," Physical Review Letters, vol. 77, no. 15, pp. 3189-3192, 1996.

[17] R. A. Smith and V. Ambegaokar, "Effect of level statistics on superconductivity in ultrasmall metallic grains," Physical Review Letters, vol. 77, no. 24, pp. 4962-4965, 1996.

[18] V. N. Gladilin, V. M. Fomin, and J. T. Devreese, "Magnetic susceptibility of ultrasmall superconductor grains," Physical Review B, vol. 70, no. 14, Article ID 144506, 2004.

[19] V. N. Gladilin, V. M. Fomin, and J. T. Devreese, "Shape of nanosize superconducting grains: does it influence pairing characteristics?" Solid State Communications, vol. 121, no. 9-10, pp. 519-523, 2002.

[20] J. Dukelsky, S. Pittel, and G. Sierra, "Colloquium: exactly solvable Richardson-Gaudin models for many-body quantum systems," Reviews of Modern Physics, vol. 76, no. 3, pp. 643-662, 2004. 

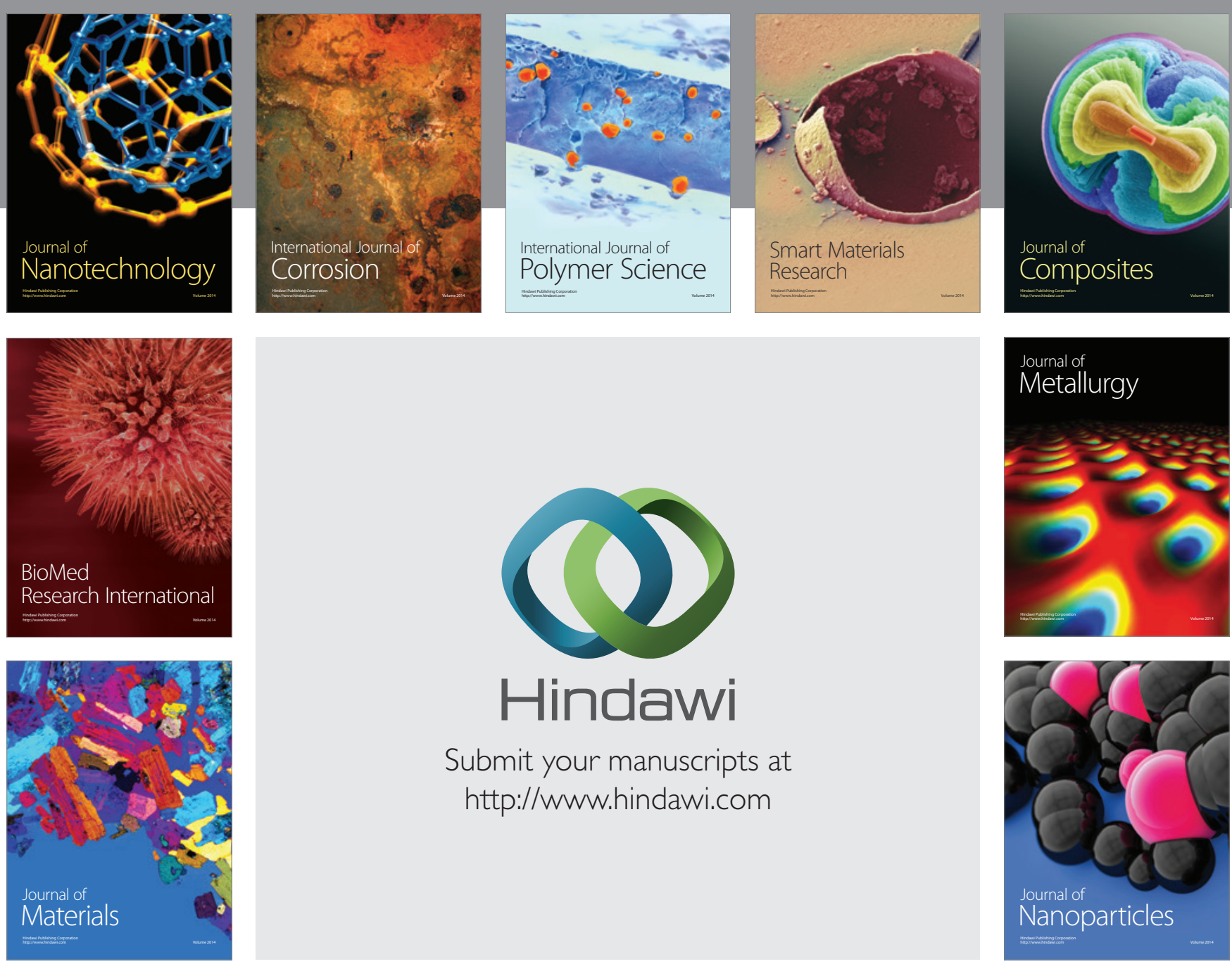

Submit your manuscripts at http://www.hindawi.com
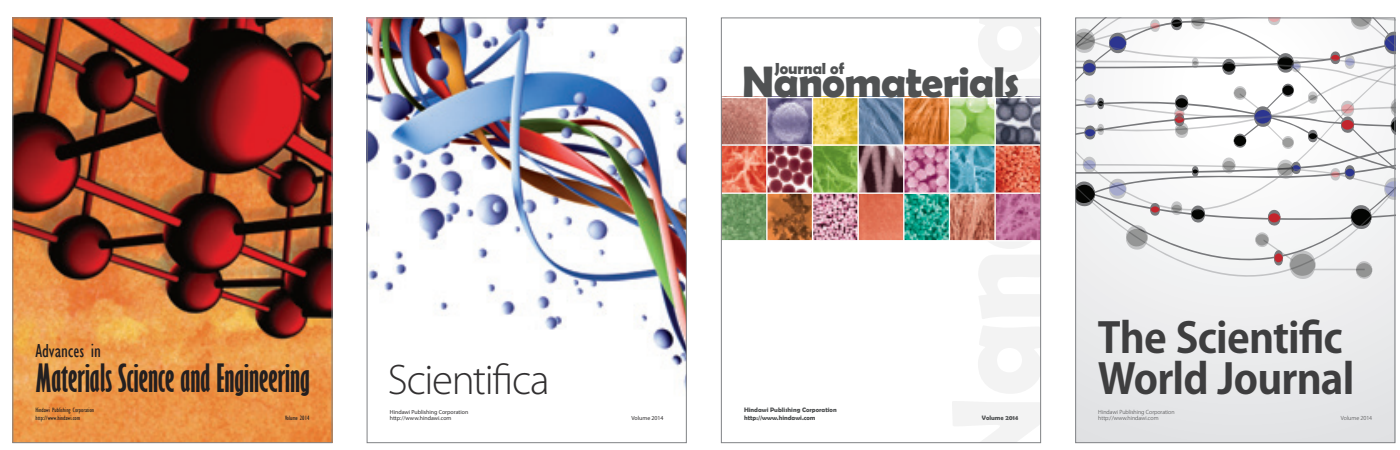

\section{The Scientific World Journal}
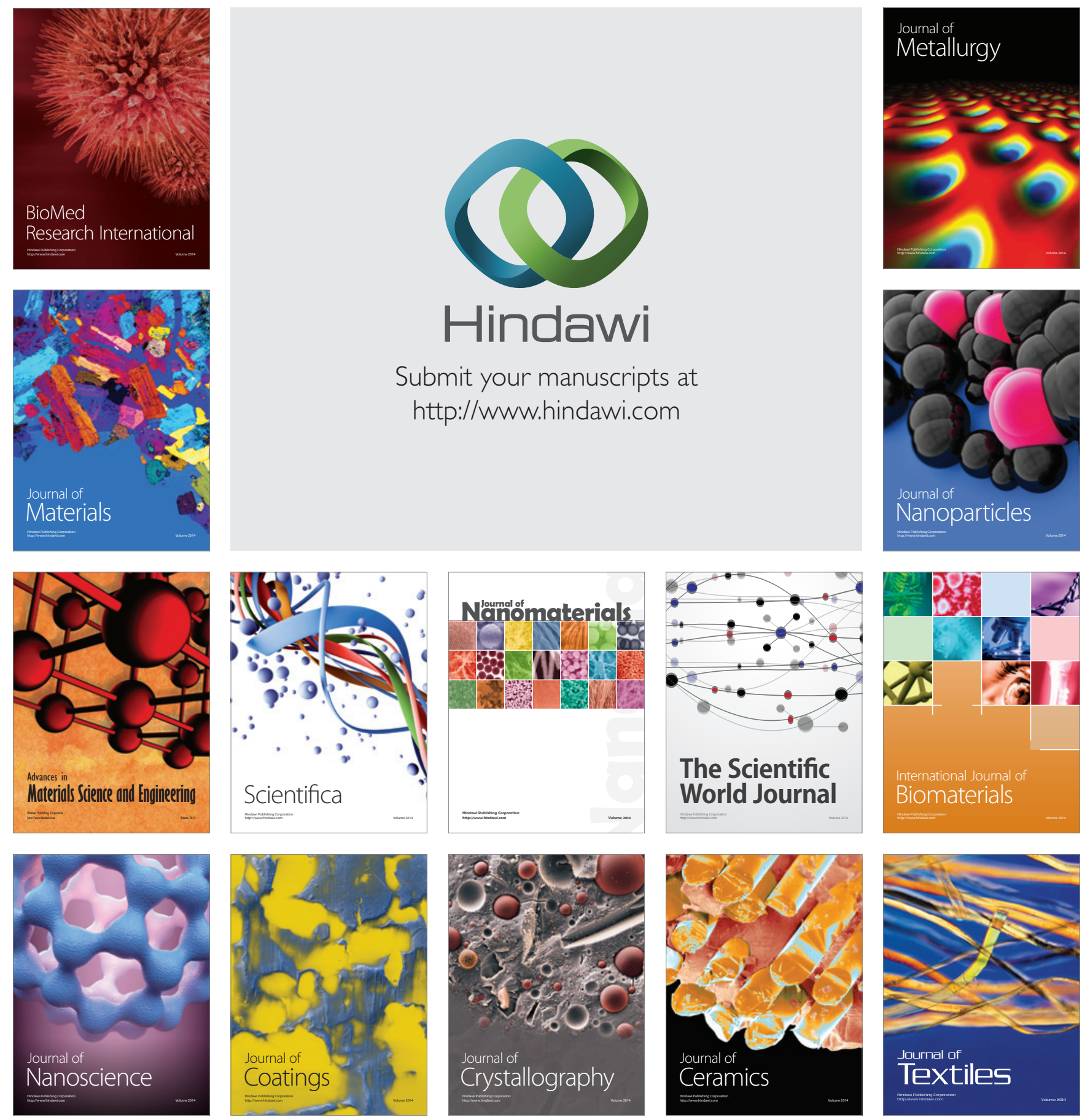